\title{
Acute Emphasematous Cholecystitis
}

\author{
Radwan Kassir ${ }^{\mathrm{a}, \mathrm{c}}$, Gabriella Ferraria, Claire Boutet ${ }^{\mathrm{b}}$, \\ Jack Porcheron ${ }^{\mathrm{a}}$
}

\begin{abstract}
Emphysematous cholecystitis (EC) is an uncommon variant of acute cholecystitis and can be rapidly lethal. We report an extremely rare case of EC and we present the imaging features of this patient. An 80-year-old male presented with epigastric pain and fever. Abdominal computed tomography confirmed the presence of an air-liquid level in the gallbladder lumen, gas within the gallbladder and in its walls. Emergency open cholecystectomy revealed necrotic changes in the gallbladder. The patient's postoperative course was uneventful. The mortality associated with non-emphysematous is $4 \%$ compared with $15 \%$ for EC due to the increased incidence of gallbladder wall gangrene and perforation. The pathophysiology of EC differs from that in acute calculous cholecystitis. Diagnosis is established when the CT scan reveals gas within the gallbladder or in its walls in the absence of an abnormal communication between the gastrointestinal tract and the biliary system. The standard treatment is emergent cholecystectomy and antibiotic therapy. It could conclude that surgeons should be aware of the existence of this kind of atypical presentation of cholecystitis. EC is a rare condition in which the abdominal computed tomograph proves to be a most useful tool. It is important to differentiate this rare pathologic feature of the gall bladder from other cholecystitis as the treatment is surgical rather than medical.
\end{abstract}

Keywords: Gall bladder; Cholecystitis; Emphysematous; CT scan; Surgery; Management

Manuscript accepted for publication December 20, 2013

\footnotetext{
${ }^{a}$ Department of Digestive Surgery, CHU Hospital, Jean Monnet University, Saint Etienne, France

${ }^{\mathrm{b}}$ Department of Radiology, CHU Hospital, Jean Monnet University, Saint Etienne, France

${ }^{\mathrm{c}}$ Corresponding author: Radwan Kassir, Department of Digestive Surgery, CHU Hospital, Jean Monnet University, Avenue Albert Raimond, 42270 Saint Etienne, France.

Email: Radwankassir42@hotmail.Fr
}

doi: http://dx.doi.org/10.14740/jcs216w

\section{Introduction}

Emphysematous cholecystitis (EC) is an uncommon variant of acute cholecystitis and can be rapidly lethal. EC is characterized by the presence of intramural and/or intraluminal gas and is a consequence of a primary ischemic injury. An early diagnosis and treatment are required.

\section{Case Report}

We report an extremely rare case of EC and we present the imaging features of this patient. An 80-year-old male presented with epigastric pain and fever. He had a history of diabetes mellitus. He presented with vomiting and acute abdominal syndrome. Clinical examination and laboratory assessment suggested acute cholecystitis. Abdominal computed tomography confirmed the presence of an air-liquid level in the gallbladder lumen, gas within the gallbladder and in its walls (Fig. 1,2). The CT scan revealed no biliary obstruction or bile duct stones. Emergency open cholecystectomy

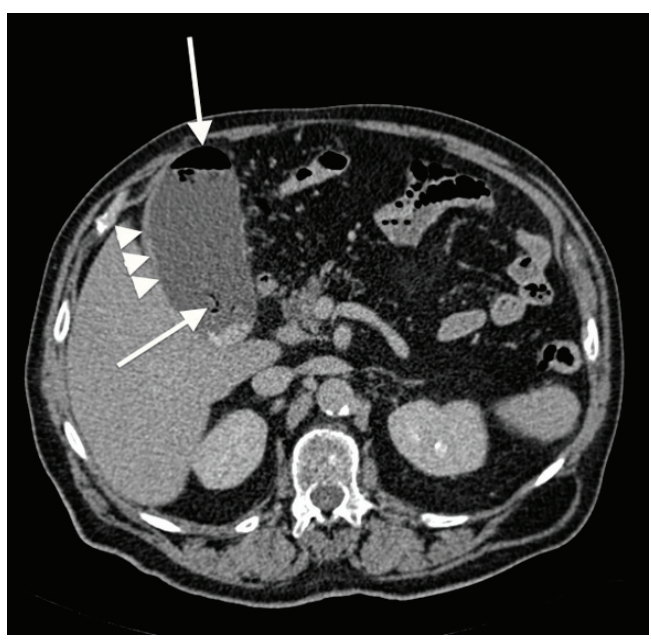

Figure 1. CT scan (axial axis) shows air in the gall bladder lumen with an air-fluid level (arrow) and gall bladder wall thickening (arrowhead). 


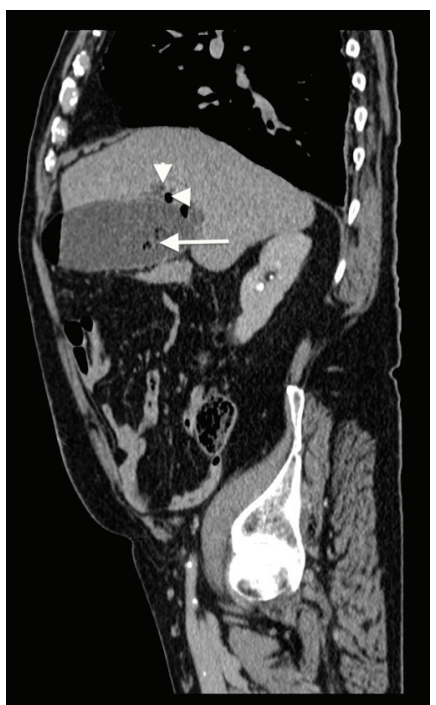

Figure 2. CT scan (sagittal axis) shows air in the gall bladder lumen (arrow) with intramural air (arrowhead) without cholelithiasis or intrahepatic abscess.

revealed necrotic changes in the gallbladder. No gallstones were detected in the gallbladder. Antibiotic treatment was given pre and post-operation. The patient's postoperative course was uneventful.

\section{Discussion}

EC is first described by May and Strong in 1971 [1]. EC is a rare variant and a life-threatening form of acute cholecystitis caused by ischemia of the gallbladder wall. CE is more common in patients with diabetes mellitus [2] and is an uncommon occurrence in the children [3]. The mortality associated with non-emphysematous is $4 \%$ compared with $15 \%$ for EC due to the increased incidence of gallbladder wall gangrene and perforation [2].

The pathophysiology of EC differs from that in acute calculous cholecystitis. The ischemia of the gallbladder wall allows mural translocation of gas-forming bacteria. Causative organisms previously reported include Clostridium perfringens, Clostridium welchii, Klebsielles, E. coli, and anaerobic Streptococci [4]. The bacteria most frequently cultured are Clostridia then Escherichia coli.

For identification of air within the biliary tract, several imagings can be used: plain radiography, ultrasound and CT. Ultrasonography demonstrates a characteristic "effervescent gallbladder". CT scan is the most sensitive and specific [5]. Diagnosis is established when the CT scan reveals gas within the gallbladder or in its walls in the absence of an abnormal communication between the gastrointestinal tract and the biliary system. Miyahara et al described a case of EC with massive gas in the abdominal cavity. In our case, the CT scan does not reveal gas within the abdominal cavity [6].

EC is associated with a higher incidence of complications in comparison with acute cholecystitis [7]. DelgadoPlasencia et al concluded that should be considered as a possible cause of pneumomediastinum [8]. EC should be considered as a possible cause of soft tissue gas gangrene [9]. Simultaneous occurrence of EC and emphysematous pancreatitis is an exceptional and potentially fatal combination [10].

The base of treatment of this disease includes: cholecystectomy, drainage, appropriate antibiotic treatment and hyperbaric oxygenation [11]. The standard treatment is emergent cholecystectomy and antibiotic therapy. EC can be safely treated by the laparoscopic approach [4]. In our case, we realized open cholecystectomy. In severely ill patients, the standard treatment is emergent percutaneous cholecystostomy with broad-spectrum antibiotics [12]. Percutaneous cholecystostomy should be considered as a possible cause of musculoskeletal complications [13].

\section{Conclusion}

EC should be considered as a possible cause of acute abdominal syndrome, especially in diabetic patients. Prompt abdominal imaging is essential in making the correct diagnosis. EC requires aggressive medical and surgical treatment. Surgeons should be aware of the existence of this kind of atypical presentation of cholecystitis.

\section{Conflict of Interest Statement}

No.

\section{References}

1. May RE, Strong R. Acute emphysematous cholecystitis. Br J Surg. 1971;58(6):453-458.

2. Moanna A, Bajaj R, del Rio C. Emphysematous cholecystitis due to Salmonella derby. Lancet Infect Dis. 2006;6(2):118-120.

3. Pal K. Laparoscopy in the management of emphysematous cholecystitis and secondary appendicitis in an 11-year-old child with insulin-dependent diabetes mellitus. Afr J Paediatr Surg. 2011;8(2):211-214.

4. Bouras G, Lunca S, Vix M, Marescaux J. A case of emphysematous cholecystitis managed by laparoscopic surgery. JSLS. 2005;9(4):478-480.

5. Grayson DE, Abbott RM, Levy AD, Sherman PM. Emphysematous infections of the abdomen and pelvis: a pictorial review. Radiographics. 2002;22(3):543-561.

6. Miyahara H, Shida D, Matsunaga H, Takahama Y, Miyamoto S. Emphysematous cholecystitis with massive 
gas in the abdominal cavity. World $\mathrm{J}$ Gastroenterol. 2013;19(4):604-606.

7. Sunnapwar A, Raut AA, Nagar AM, Katre R. Emphysematous cholecystitis: Imaging findings in nine patients. Indian J Radiol Imaging. 2011;21(2):142-146.

8. Delgado-Plasencia L, Gonzalez-Garcia I, RodriguezGonzalez D, Torres-Monzon AE. Pneumomediastinum as a complication of emphysematous cholecystitis: case report. BMC Gastroenterol. 2010;10:99.

9. Safioleas M, Stamatakos M, Kanakis M, Sargedi C, Safioleas C, Smirnis A, Vaiopoulos G. Soft tissue gas gangrene: a severe complication of emphysematous cholecystitis. Tohoku J Exp Med. 2007;213(4):323-328.
10. Choi HS, Lee YS, Park SB, Yoon Y. Simultaneous emphysematous cholecystitis and emphysematous pancreatitis: a case report. Clin Imaging. 2010;34(3):239-241.

11. Stojanovic D, Lalosevic D, Stojanovic M, Caparevic Z, Dosev M, Ilic J. [Emphysematous cholecystitis]. Med Pregl. 2002;55(11-12):529-531.

12. Wu JM, Lee CY, Wu YM. Emphysematous cholecystitis. Am J Surg. 2010;200(4):e53-54.

13. Safioleas M, Stamatakos MK, Mouzopoulos GJ, Tziortzis G, Chagiconstantinu K, Revenas K. Emphysematous cholecystitis. Review of five cases and report of septic musculoskeletal complications. Chirurgia (Bucur). 2006;101(1):61-64. 